\title{
Word length and frequency as determinants of stuttering'
}

I. M. SCHLESINGER, RACHEL MELKMAN, AND RON LEVY

HEBREW UNIVERSITY, JERUSALEM

Word length and Frequency were independently varied to assess their effect on stuttering frequency. The list of test words contained words of one, two or three syllables which were of low, medium of high frequency of occurrence. Stuttering frequency of 31 young stutterers increased significantly with word length and decreased significantly with frequency of occurrence.

Stuttering frequency in reading a text has been shown to decrease with transitional probabilities as estimated by word-by-word guessing, as well as with frequency of occurrence of the word (Schlesinger et al, 1965). In the latter study, however, word frequency was confounded with word length. The present study was aimed at isolating the latter variables so as to study their effect on stuttering. It was hypothesized that stuttering frequency would increase with word length and decrease with word frequency.

Subjects

Thirty-one stutterers, 20 boys and 11 girls, were tested. The age range was $8-16$ years.

\section{Materials and Procedure}

Three degrees of word frequency and three word lengths were varied independently in constructing a list of Hebrew test words. Word frequency was determined by Rieger's (1935) Hebrew word count. Three degrees of word frequency were employed: words appearing in the first third of his list of 2,017 most frequent words, words appearing in the last third of the list, and words not included in the list (because they were not frequent enough). Words one, two and three syllables long were employed. Five test words were included in each of the nine groups determined by these three degrees of frequency and three word lengths. In each group the five words had the same five initial sounds: $/ \mathrm{m} /, / 1 /, / \mathrm{k} /$, $/ \mathrm{a} /$, and $/ \mathrm{e} /$.

It was intended, initially, to include also four-syllable words. In Hebrew, however, such words are rare, unless in inflected form. To obtain additional information, inflected words with four syllables were included in the test list. Five high-frequency and five mediumfrequency inflected words were included (beginning with the above initial sounds), but for the low-frequency group only four such words could be found. Since inflected words are not strictly comparable to the other, uninflected words of the list, they were not included in the analyse in testing the hypothesis.

The 59 test words were arranged in random order with the following constraint: when the list was divided into groups of four consecutive words, each such group contained one word of each length.
The test words were typed one above the other in three columns with five arbitrarily chosen words of different lengths preceding them and another five following them.

An additional list of 15 words was prepared which was read by $\mathrm{S}$ prior to reading the test list, so that he would adapt to the experimental situation. Next, he read the test list three consecutive times.

\section{Results and Discussion}

Since comparisons had to be made between words of different lengths, only stutterings on the first syllable were taken into account in the analysis. A total of 481 words were stuttered on the first syllable by the $31 \mathrm{Ss}$ on three readings. Table 1 gives a breakdown of these stutterings by word frequency and word length.

As shown in Table 1, both experimental variables affect stuttering frequency, the number of words stuttered being about twice as large for low-frequency words than for high-frequency words, and about 3-1/2 times as large for three-syllable words than for onesyllable words. Each of the effects was significant at the .001 level by a Friedman two-way analysis of variance (Siegel, 1956).

The number of stutterings on the first syllable of four-syllable words also increased with frequency of occurrence: 84 (for four words only), 80 , and 175 . These numbers are higher than those for three-syllable words.

The obtained effect of word frequency may be explained as being due to response strength (Schlesinger et al, 1965). To explain the effect of word length, one might assume that, through past experience, longer words have become associated with difficulty in articulation. At any rate, the present results are in line with those obtained in other areas, showing that word length affected performance independently of frequency in satiation of meaning (Wertheimer \& Gillis, 1958), and tachistoscopic recognition (Newbigging, 1961), rate of reading (Pierce \& Karlin, 1957), and language disorders (Filby et al, 1963).

Table 1. Number of stutterings in three readings by word frequency and length. ( $N=31$ )

\begin{tabular}{lcccr} 
Length & \multicolumn{4}{c}{ Frequency } \\
\hline & High & Medium & Low & Total \\
\hline One syllable & 18 & 23 & 28 & 69 \\
Two syllables & 39 & 50 & 61 & 150 \\
Three syllables & 58 & 69 & 135 & 262 \\
\cline { 2 - 5 } Total & 115 & 142 & 224 & 481 \\
\hline
\end{tabular}




\section{Roferences}

Filby, Y., Edwards, A. E., \& Seacat, G. F. Word length, frequency and similarity in the discrimination behavior of aphasics. $J$. speech hear. Res., 1963, 6, 255-261.

Newbigging, P. L. The perceptual redintegration of frequent and infrequent words. Canad. J. Psychol., 1961, 15, 133-142.

Pierce, J. R., \& Karlin, J. E. Reading rates and the information rate of a human channel. Bell Sys. tech. J., 1957, 36. 497-516.

Rieger, E. Otzar Milot Hayessod (list of Basic Words in Hebrew). Jerusalem: Hebrew Teacher's Seminary, 1935.
Schlesinger, I. M., Melkman, R., Forte, M., \& Fried, B. Stuttering, information load, and response strength. J. speech hear. Dis., $1965,30,32-36$.

Siegel, S. Nonparametric Statistics for the Behavioral Sciences, New York: McGraw Hill, 1956.

Wertheimer, M., \& Gillis, W. M. Satiation and the rate of lapse of verbal meaning. J. gen. Psychol., 1958, 59, 79-85.

\section{Note}

1. We are grateful to Mrs. Lilly Tell of the Audiology Center of the Hadassah Medical Center, Jerusalem, for her cooperation, and to Dr. Charles W. Greenbaum for his comments on a draft of this paper. 